\title{
Fuzzy logic expert system for evaluating the activity of university teachers
}

\author{
Vasile Florin Popescu ${ }^{(1)}$, , Marius Sorin Pistol (iD)2
}

\author{
${ }^{1}$ National Defense University, Faculty of Security and Defense, Dep. Information Systems and Cyber Defense \\ Romania \\ ${ }^{2}$ European Commision, European Asylum Support Office, Malta
}

\begin{abstract}
ARTICLE HISTORY
Received: Mar. 09, 2020

Revised: Oct. 31, 2021

Accepted: Nov. 21, 2021

Keywords:

Fuzzification,

Defusification,

Inference,

Mamdani,

Natural language.

Abstract: Assessing the performance of academics at different levels is increasingly difficult to achieve using traditional methods based mainly on numerical scores in evaluating teaching and research activity. The indexing of academic performance in various international databases with impact indices at different scales has led to the need for advanced computer models, such as expert systems based on fuzzy logic, proposed in this research, which address the evaluation of teachers even in the face of imprecise information and under conditions of uncertainty. In this research, as a contribution and novelty, a fuzzy logic model was developed in which an algorithm was simulated and implemented in Matlab using the Mandami toolkit, which allows inference of the rules of fuzzy logic and visualization. 3D. The system implementation was done by software in Matlab environment, using systems with fuzzy Mandami logic. The result of this pilot study was to test and validate the proposed model through a graphical interface, giving the results according to minimum criteria and with additional explanations.
\end{abstract}

\section{INTRODUCTION}

Fuzzy systems are an alternative to the traditional methods of dealing with affiliation and logic, which have their origins in ancient Greek philosophy and applications in the field of artificial intelligence. Despite its long-lived origins, it is a relatively new field and therefore there is still plenty of room for research. The application of fuzzy logic as a simple method for deciding on an unambiguous evaluation of university teachers based on ambiguity, vagueness, imprecision, or lack of input information requires several numerical parameters to work in terms of what is considered "significant error" and "error variation," but the exact values of these numbers are not critical unless good performance is required. Fuzzy logic does not require very precise numerical inputs, in terms of evaluating university teachers, is inherently robust, and can handle any reasonable number of inputs, but the complexity of the system increases significantly with the number of inputs and outputs. Rules based on simple language, such as IF X and Y THEN $Z$, are used to describe the desired response of the system in terms of linguistic variables rather than mathematical formulas. Their number depends on the number of inputs and outputs and the goals of the designer in controlling the response. Fuzzy systems, including fuzzy logic and

\footnotetext{
*CONTACT: Popescu Florin $\rrbracket$ popescuveve@gmail.com $\equiv$ National Defense University, Faculty of
} Security and Defense, Dep. Information Systems and Cyber Defense, Romania 
fuzzy set theory, represent a rich and important extension of standard logic used in higher education assessment. The mathematics developed based on these theories is consistent, and fuzzy logic can be a generalisation of classical logic. Applications that can be generated from or adapted to fuzzy logic are widespread and provide the ability to model conditions that are vaguely defined despite the concerns of classical logicians. Many systems can be modelled, simulated and even physically implemented using fuzzy systems, such as the present study.

On many websites, in many scientific articles, or in talks at scientific symposia and conferences, many of us have read or heard terms such as artificial neural networks, fuzzy logic, genetic algorithms, genetic programming, evolutionary computation, expert systems, gravity algorithm, ant algorithm, particle group optimization, multiagent systems, and others. All of these terms are concepts that describe various methods and techniques for solving problems of moderate and high complexity based on the simulation of intelligent behavior, and they are grouped under the umbrella of two terms that sound similar but usually refer to different things: Artificial Intelligence and Intelligent Computing. Although there are supporters of the idea that the two terms actually refer to the same thing, most opinions hold that Artificial Intelligence and Intelligent Computing, while having similar goals, have fundamental elements that distinguish them. Artificial intelligence (AI) is the older of the two terms. The term was first used in its current meaning in 1956 at a scientific symposium at Dartmouth College in Hanover, USA. The Father IA, John McCarthy, defined it as "the science and technology of creating intelligent machines" (McCarthy, 1959) in the form of hardware or software. One of the most commonly cited definitions is that of the study and development of intelligent agents, where an intelligent agent is understood to be an autonomous entity that observes and interacts with the environment in an attempt to achieve goals.

The second term, Intelligent Computing (CI) or Computational Intelligence, was first used in 1990 by the IEEE Neural Networks Council, founded ten years earlier, which became a IEEE society in 2001 and later changed its name to IEEE Computational Intelligence Society to include new areas of interest such as fuzzy systems and evolutionary computing. The field of AI can be defined as a collection of natural inspiration techniques and computational methods that are distinct from the traditional techniques associated with AI and intended for the creation of intelligent systems. This collection includes subfields such as artificial neural networks, fuzzy systems, evolutionary computation, machine learning, Bayesian reasoning and so on. The core technologies of IC include artificial neural networks (RNA), fuzzy systems (SF), and evolutionary computation (EC), as well as hybrid intelligent systems that incorporate these technologies and other related paradigms. A taxonomy of intelligent systems, with the main research areas and components that define them, is shown in Figure 1.

Sources of uncertainty:

Imperfection of the rule

Uncertainty of the evidence

$\checkmark$ Confidence in the conclusion must be scaled

$\checkmark$ Use of vague, imprecise language

Ways to express uncertainty:
$\checkmark$ Probabilities
$\checkmark$ Fuzzy logic
$\checkmark$ Bayes' theorem
$\checkmark$ Dumpster-Shafer theory 
Figure 1. Intelligent systems.

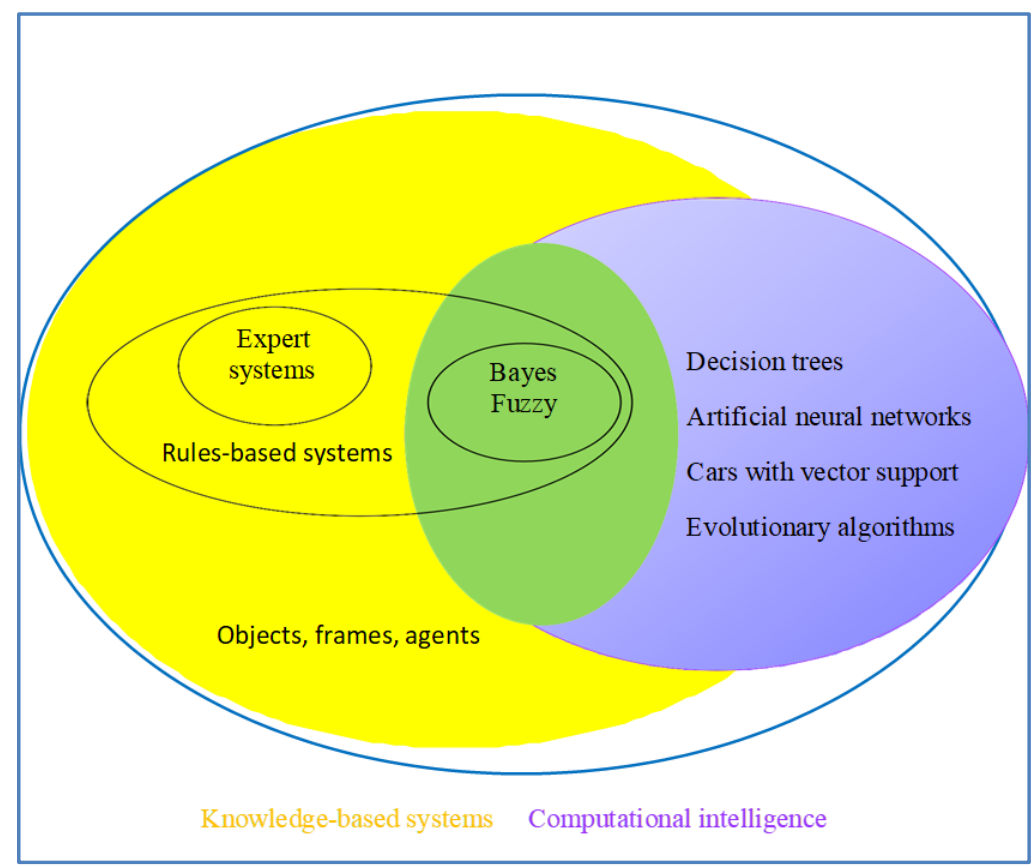

Reasoning techniques in unsafe environments:

Bayesian theory - probabilistic method

Certainty theory

Theory of possibility (fuzzy logic)

Heuristic Methods

The possibility theory - a short history

Parmenides (400 B.C.)

Aristotle

- "Law of the Excluded Middle" - every sentence must be True

or False

$\checkmark$ Plato

- the third region between True and False

- Lay the groundwork for fuzzy logic

Lukasiewicz, \& Tarski (1930)

- Proposes a systematic alternative to Aristotle's bivalent logic - trivalent logic: True, False, Possible

$\checkmark$ Zadeh, L. (1965).

- Mathematically describes the theory of fuzzy sets and fuzzy logic: the membership function (True and False values) operates on the interval $[0,1]$

- Proposed new computational operations for fuzzy logic

- He considered fuzzy logic a generalization of classical logic

- Published the first article about fuzzy crowds

When is it important to use fuzzy logic systems?

Queries in natural language

$\checkmark$ Representation of knowledge in expert systems

$\checkmark$ Fuzzy control - when working with inaccurate phenomena (disturbed by noise) 


\section{METHOD}

The model of fuzzy logic applied to the evaluation of university professors is an approximation method that can be used to formally model vague "knowledge" stored in a base of rules. The application of fuzzy logic in the evaluation of university employees at different levels is due to the advantages it offers in the following specific situations:

- Enables modelling of non-linear, complex, or imprecisely known processes for evaluation of university personnel, according to level;

- allows the implementation of the human experience of the evaluators, in this case in the construction of the inference rules, using the linguistic variables explained in the theoretical part.

\subsection{Brief Description of the Concept of Fuzzy Logic, Mandami Model}

The theoretical foundations of fuzzy logic were laid in 1965 by Lofti A. Zadeh, a professor at Berkely University in California. The term fuzzy logic was introduced by Zadeh at the same time as the proposal of fuzzy sets, but elements of fuzzy logic have been studied since 1920 (Garrido, 2012), reminiscent of the work of Łukasiewicz and Tarski, in which the so-called nvalued logic is proposed. However, the logic and fuzzy sets as they are known and used today are those proposed in the research of Zadeh.

In classical mathematics, an element is part of a set or not, whether it belongs to that set or not. In other words, the membership of elements in a given set is treated on a binary basis. Zadeh's theory of fuzzy sets, on the other hand, defines classical sets and their associated values in terms of crisp. Moreover, the new theory offers the possibility to evaluate step by step the membership of an element to a set by quantifying it using the so-called membership functions, which take values in the range $[0,1]$.

In fuzzy logic, the discrete values of Boolean logic (false and true) are replaced by a continuous membership function that takes values in the range [0,1], where 0 stands for absolutely false and 1 for absolutely true. Consequently, an imprecise formulation has an associated truth value between 0 and 1 .

For the new fuzzy sets, it was necessary to define the elementary operations for which it was proposed to use the complement against 1 for negation, the max operator for union and the min operator for intersection (Zadeh, 1996). Then fuzzy numbers, elementary algebraic operations with fuzzy numbers, fuzzy intervals and relations between fuzzy quantities were defined. Moreover, in order to maintain the connection with natural language and to allow a simple mathematical representation, the notion of modifier or qualifier, an equivalent to adjectives or adverbs in grammar, was introduced; thus, qualifiers are used such as: close, very, extremely, possible, with certainty, and so on.

The following are some important moments in the history of fuzzy systems. After the first moment in 1965, Professor Zadeh proposed the use of fuzzy algorithms in 1968 (Zadeh, 1968) and fuzzy decision systems in 1970 (Bellman \& Zadeh, 1970). In 1971, he published the work Quantitative Fuzzy Semantics (Zadeh, 1971), in which he proposed the formal elements on the basis of which the methodology and the various types of applications of fuzzy logic were later developed. In 1973, he published a reference work (Zadeh, 1973) in which he defined linguistic variables and IF-THEN rules for the formation of knowledge bases. The first fuzzy controller for controlling an engine and a steam boiler was designed by Ibrahim Mandani in the mid-1970s (Mamdani \& Assilian, 1975).

1987 is the year when the first commercial applications for different types of fuzzy controllers are developed and built, such as the fuzzy controller developed by Hitachi for the famous Japanese train Sendai, or those developed by Omron, another Japanese company that developed 
the fastest fuzzy controller or the first fuzzy chip for microcomputers SUGE. Later, in 1993, the first application of fuzzy logic was registered for controlling a water treatment plant - also in Japan, of course.

In the mid-1980s, there were the first attempts at the theoretical foundation and practical development of fuzzy control systems based on fuzzy data sets combined with fuzzy learning. The foundations of fuzzy control systems are due to Professors Tomohiro Takagi of Meiji University (Tokyo) and Michio Sugeno of Doshisha University (Takagi \& Sugeno, 1985).

Since the 1990s, applications of crowds and fuzzy logic in daily life have become more present and have developed rapidly. Numerous applications of this type can be found in the profile industry, the most famous being control systems for washing machines (Ahmed \& Toki, 2016), ABS systems for braking (Subbulakshmi, 2014), autofocus systems for video cameras, elevator control systems (Patjoshi \& Mohapatra, 2010) or philtres against spam messages (Vijayan et al., 2011).

The realisation of a flexible method for solving indeterminacy problems has been achieved through the development of fuzzy systems, which are based on fuzzy logic and are a special case of expert systems. Fuzzy logic works with the elements $A=\{x / x[0,1]\}$ and assigns a degree of belonging to the set to the object. The robustness of fuzzy logic is emphasised by the simultaneous control of numerical data and lexical knowledge (linguistic variables) by interpreting quantitative terms qualitatively.

The linguistic variable is a property and as a structure it includes (Chennakesava, 2008):

a. The linguistic value $\mathrm{u}$ is an adverb, an adjective associated with the linguistic variable, indicating the name of the associated fuzzy set;

b. The representation field $U$ is a classical set on which fuzzy sets are defined. The set $U$ is also called representation field, discourse universe or reference set;

c. The membership function $\mu \mathrm{F}$ assigns to each element $\mathrm{u}$ the degree of membership in the fuzzy set F;

d. The degree of membership $\mu$ represents the extent to which an element belongs to a fuzzy set.

In order to understand the theory of fuzzy logic and the fuzzy set, it is necessary to present the elements on which it is based (Chuen, 1990). Let $U$ be a set of objects with the general name $\{\mathrm{u}\}$, which can be discrete or continuous. $\mathrm{U}$ is called the representation domain (universe of discourse) and $u$ represents the generic elements of $U$. Denotation 1. Fuzzy set: a fuzzy set $F$ contained in the representation domain $\mathrm{U}$ is characterized by the membership function $\mu \mathrm{F}$ which takes values in the range $[0,1]$, i.e. F: $\mathrm{U}[0,1]$.

The stages of the construction of a fuzzy logic system, the Mamdani model, are shown in Figure 2. The theory of possibility, and implicitly Fuzzy Logic, is based on the following concepts:

- Generalization of Boolean logic

- Manipulates the concept of partial truth

o Classical logic - everything is expressed in binary terms

o 0 or 1 , white or black, yes or no

o Fuzzy logic - the gradual expression of a truth.

o Values between 0 and 1

The basic idea of this pilot study on the evaluation of academics using Fuzzy logic systems is:

- In accordance with the theory of certain information:

$\circ$ Florin Popescu is an associate professor 
- In accordance with the theory of uncertain information:

$\checkmark$ In accordance with probability theory:

○ There is an $80 \%$ chance that Florin Popescu will be an associate professor

$\checkmark$ In accordance with fuzzy logic:

○ The degree of affiliation of Florin Popescu to the group of university lecturers is 0.8

- Definition of inputs and outputs by the expert

o Gross input and output data

$\circ$ Fuzzification of input and output data

- Determining fuzzy variables and fuzzy sets based on membership functions

- Construction of a rule base by the expert

o Decision matrix of knowledge base

- Evaluation of rules

○ Inference - transforming fuzzy inputs into fuzzy outputs by applying rules from knowledge base

- Defuzzification

- Interpretation of the results

Figure 2. Fuzzy logic system.

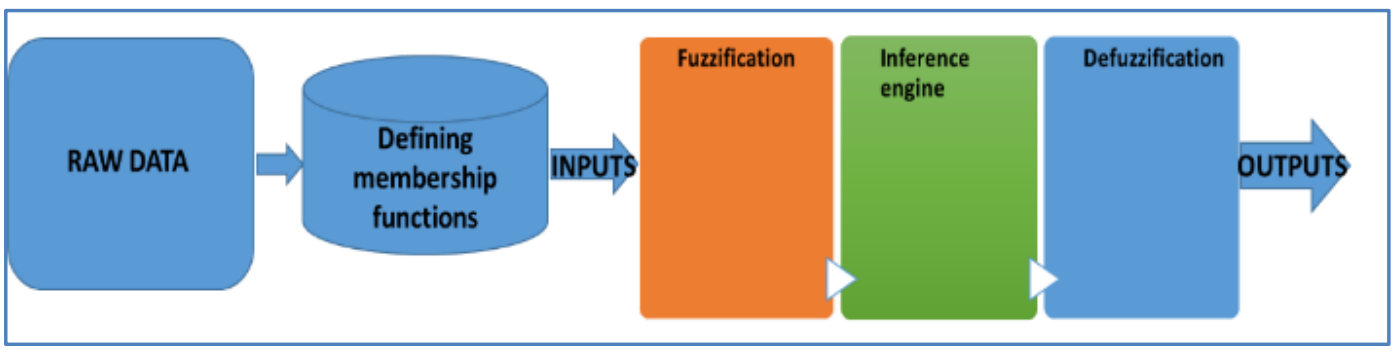

A. Fuzzification of input data

- Defining any set - 2 ways:

o By enumerating the elements

$\checkmark$ Example: Crowd of students $=\{$ John, Stann, Brown $\}$

$\circ$ By specifying a property of the set elements

$\checkmark$ Example: The set of numbers seems $=\{x \mid x=2 n$, where $\mathrm{n}$ - natural number $\}$

- The characteristic function $\mu$ of a set

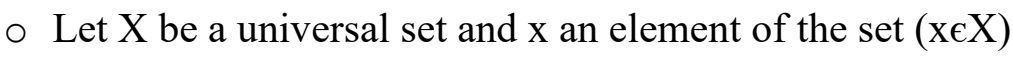

○ Classical logic

$\checkmark$ Let $\mathrm{R}$ be a subset of $X: \operatorname{Re} X, R$ - regular set

$\checkmark$ Whether or not element $\mathrm{x}$ belongs to the set $\mathrm{R}$

$\checkmark \mu_{\mathrm{R}}: X \rightarrow\{0,1\}, e_{R}(x)=1, x \in R / 0, x \notin R$

○ Fuzzy logic

$\checkmark$ Let $\mathrm{F}$ be a subset of $\mathrm{X}$ (discourse universe): $F \in X, F$ - fuzzy sets

$\checkmark$ any element $\mathrm{x}$ belongs to the set $\mathrm{F}$ in a certain degree $\mu \mathrm{F}(\mathrm{x})$

$\checkmark \mu_{F}: X \in[0,1], \mu_{F}(x)=g$, where $g \epsilon[0,1]$ degree of belonging of $\mathrm{x}$ to $\mathrm{F}$

$\checkmark g=0 \in$ not belonging

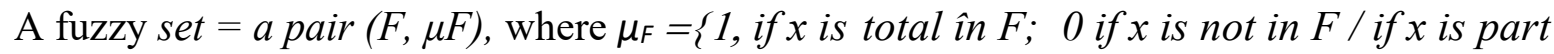
of $F$ (x fuzzy number). 


\subsection{Mamdani Model}

- Basic idea:

o a consequence of the rule is the form "output variable is part of a fuzzy set"

oThe result of the evaluation of the premises is applied for the function to which the consequence belongs.

o Example: If $\mathrm{x}$ is in $\mathrm{A}$ and $\mathrm{y}$ is in $\mathrm{B}$ then $\mathrm{z}$ is in $\mathrm{C}$.

- Typology (depending on how the result is applied to the function to which the consequence belongs)

$\circ$ Fuzzy sets result from clipped type

$\checkmark$ The function of belonging of the consequence is cut at the level indicated by the truth value of the result

Advantage: easy calculations

Disadvantage: possible information is lost

○ Fuzzy sets result of scaled type - Mamdani model

$\checkmark$ The function of belonging of the consequence is adjusted by scaling (multiplication) the truth value of the result

Advantage: less information is lost

$>$ Disadvantage: more complicated calculations.

Fuzzy Logic Toolbox ${ }^{\mathrm{TM}}$ software supports two types of fuzzy inference systems:

- Mamdani systems

- Sugeno systems

\subsubsection{Mandami inference used}

For simulation, modeling and validation of the results, 2 inference models were used in this research: inference (max-min) with a single rule and with multiple rules.

\subsubsection{Mandami inference (max-min) with a single rule.}

Modus Ponens generalized:

In fuzzy logic and rough reasoning, the most important rule of inference is generalized Modus Ponens.

In classical logic, this rule of inference is form $(p \wedge(p \rightarrow q)) \rightarrow q$, namely:

rule: if $p$, then $q$

premise: $p$

conclusion: $q$

In fuzzy logic, the corresponding inference rule is as follows:

rule: if $x$ is $A$, then $y$ is $B$

premise: $x$ is $A^{\prime}$

conclusion (consistent): and this $B^{\prime}$

If $\mathrm{A}^{\prime}=\mathrm{A}$ and $\mathrm{B}^{\prime}=\mathrm{B}$, the rule is reduced to classical Modus Ponens. The matrix $\mathrm{A} \rightarrow \mathrm{B}$ is often denoted by $R$. The fuzzy inference process is seen as a transformation of one fuzzy set into another fuzzy set. The subset induced in B, B 'is calculated as follows:

$$
b_{j}{ }^{\prime}=\max \left(\min \left(a_{i}{ }^{\prime}, r_{i j}\right)\right) \text {. }
$$

There are several methods for defining the matrix R. In the following, we will use the Mandami inference type, whereby the set B 'is a "cut" variant of B, at the height set by A' (see Figure 3). $A$ 'can be a normal subset of $A$, not just a single element. 
Figure 3. Mandami type inference.

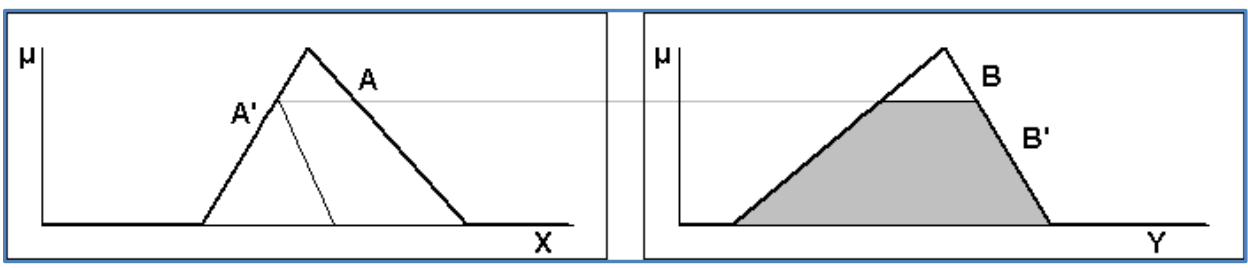

Defuzzification:

After determining the fuzzy set induced by an inference rule, in some applications a singular strict value must be determined based on this set. This process is called defuzzification. The most commonly used defuzzification technique is the centroid method (or centroid method):

$$
x_{C G}=\frac{\sum_{i} x_{i} \cdot \mu_{A}\left(x_{i}\right)}{\sum_{i} \mu_{A}\left(x_{i}\right)}
$$

In Figure 4, the program uses intervals $(0,100)$ as universes of discourse. In the figure on the right, the blue bounded set is $\mathrm{B}$, the pink bounded set is $\mathrm{B}$ 'and the red marked value on the $\mathrm{X}$ axis is the centroid.

Figure 4. Mamdani type inference and defuzzification.

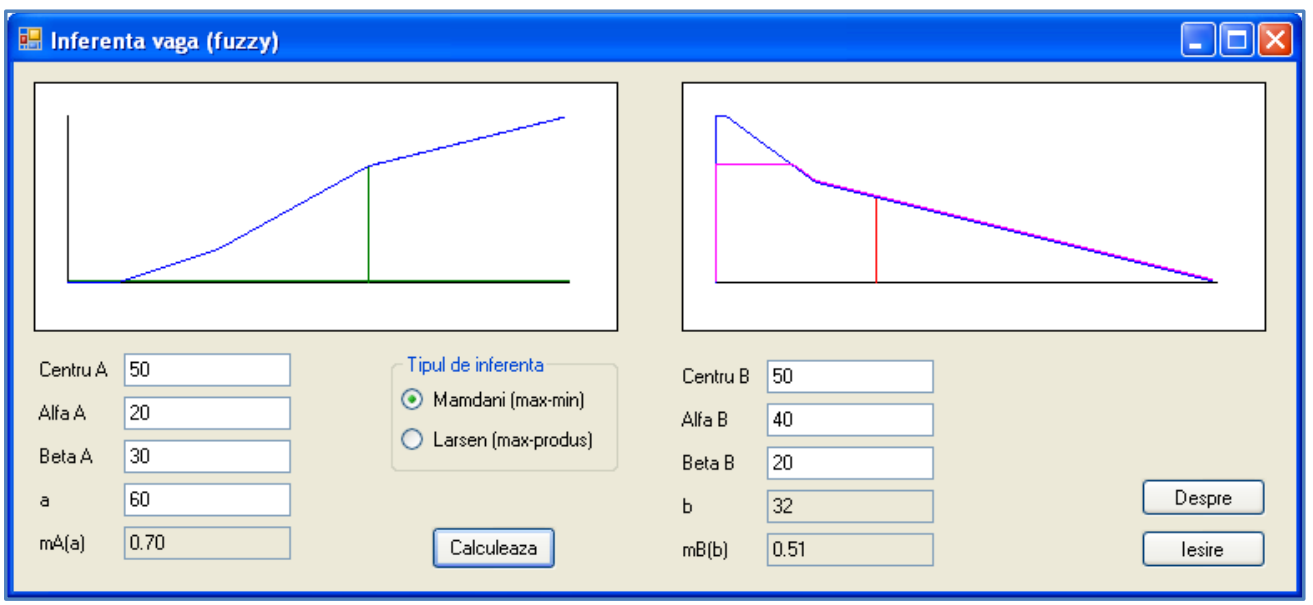

2.2.1.2. Mandami inference with multiple rules. An example of a Mandami-type fuzzy inference system is shown in Figure 5. To calculate the output of this system when the inputs are given, the following 6 steps must be followed:

1. It determines a lot of fuzzy rules;

2. The fuzzification of the entries is performed using the membership functions;

3. Combine the fuzzy inputs following the fuzzy rules to establish the activation powers of the rules;

4. Calculate the consistency of the rules by combining the activating powers of the rules with the membership functions of the outputs;

5. Combine consistencies to determine the output set;

6. Defuzzify the output set only if you want the output to be strict. The following is a detailed description of this process. 
Figure 5. Mandami type fuzzy inference system with two rules and two strict inputs.

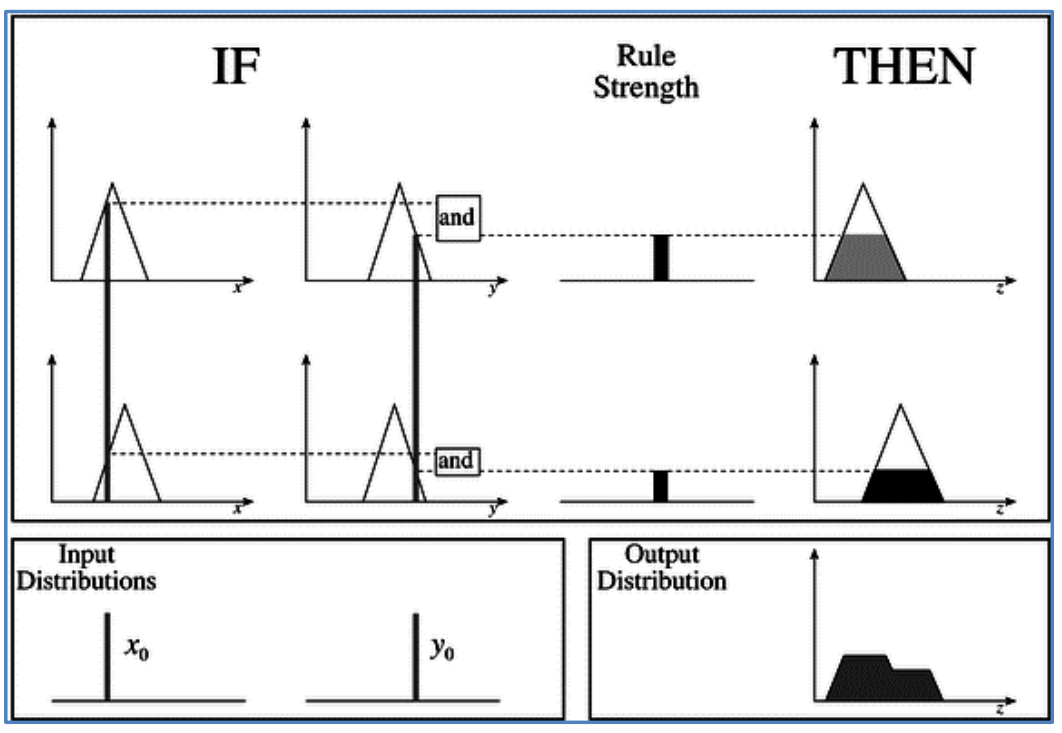

\section{Creating fuzzy rules}

Fuzzy rules are a set of statements that describe how the system can make a decision about estimating the output. Fuzzy rules have the following form: IF (input-1 is set-fuzzy-1) AND / OR (input-2 is set-fuzzy-2) AND / OR... THEN (output is set-fuzzy-output).

An example of a rule written in this way is the following: IF the project funding is sufficient AND the number of employees is low THEN the project risk is low. In the example in Figure 6 , there are two inputs $\mathrm{x} 0$ and $\mathrm{y} 0$ shown in the lower left corner. For these strict inputs, the degrees of affiliation are marked in the corresponding sets. Combining Multiple Antecedents When creating fuzzy rules, we use the operators AND, AND and sometimes NEGATION. The fuzzy operator AND is written as follows: $\mathrm{AB}(\mathrm{x}) \mathrm{T}(\mathrm{A}(\mathrm{x}), \mathrm{B}(\mathrm{x}))$, where $\mathrm{T}$ is a function called the T-norm, $\mu \mathrm{A}(\mathrm{x})$ is the degree to which $\mathrm{x}$ belongs to set $\mathrm{A}$, and $\mu \mathrm{B}(\mathrm{x})$ is the degree to which $\mathrm{x}$ belongs to set $\mathrm{B}$. Although there are several ways to compute the function AND, the most commonly used is: $\min (\mu \mathrm{A}(\mathrm{x}), \mu \mathrm{B}(\mathrm{x}))$.

The fuzzy operator AND is a generalization of the Boolean logical operator AND in the sense that the truth value of a proposition is not just 0 or 1 , but can be between 0 and 1 . A T-norm function is monotone, commutative, associative and observes the conditions $T(0,0)=0$ and $\mathrm{T}(\mathrm{x}, 1)=\mathrm{x}$.

The fuzzy operator OR is written as AB S(A, B), where $\mathrm{S}$ is a function called T-corma. Similar to the AND operator, this can be: $\max (\mu \mathrm{A}(\mathrm{x}), \mu \mathrm{B}(\mathrm{x}))$. The fuzzy operator OR is also a generalization of the Boolean logical operator OR to values between 0 and 1 . A T-conorm function is monotonic, commutative, associative, and obeys the conditions $\mathrm{S}(\mathrm{x}, 0)=\mathrm{x}$ and $\mathrm{S}$ $(1,1)=1$.

Calculation of consistencies:

First, the activation forces of the rules are calculated, as described earlier. In Figure 6, it can be seen that the fuzzy operator AND is applied to the membership functions to calculate the activation forces of the rules.

Then, for a Mandami type fuzzy inference system, the output set is truncated at the level given by the activation force of the rule, as shown in the previous sections.

Aggregation of outputs:

The outputs obtained after applying the fuzzy rules are combined to obtain the output set. This is usually done using the fuzzy operator OR. In Figure 6, the membership functions on the right 
are combined with the fuzzy operator OR to obtain the output set shown in the lower right corner.

Defuzzification:

It is often desirable to get a strict outcome. For example, if you are trying to classify handwritten letters on a blackboard, the fuzzy inference system must produce a strict number that can be interpreted. This number is obtained after the defuzzification process. The most commonly used defuzzification method is the Center of Gravity method, which is illustrated in Figure 6.

Figure 6. Defuzzification using the center of gravity method.

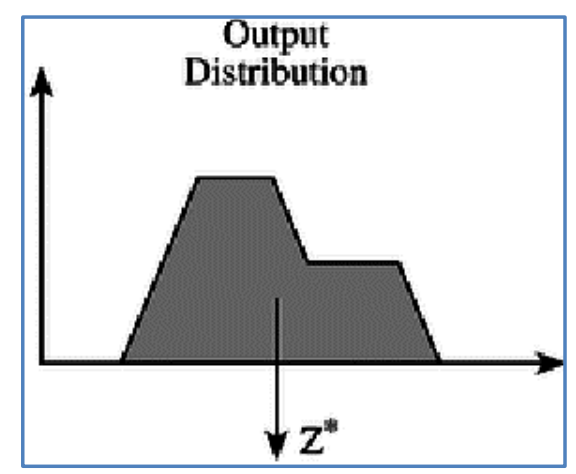

\subsection{Software Implementation of Fuzzy Algorithms}

For the moment, we understand an algorithm to be a general method for solving a particular type of problem that can be implemented on a computer. In this context, an algorithm is the absolute essence of a routine. The algorithm underlying the fuzzy automaton contains an algebraic part in addition to the logical part. Thus, it is a mixed type algorithm organized as a finite sequence of steps and includes several specific operations. They meet the basic requirements to be implemented on the computer, that is, they are defined and effective. According to the famous equation of N. Wirth:

$$
\text { algorithms }+ \text { data structures }=\text { programs }
$$

A program consists of a set of procedures, which are considered black boxes, and an associated set of data on which the procedures operate. The form that the algorithm takes in a computer implementation is subordinate to the programming style and depends in particular on the type of language. Figure 2 shows the logical scheme of the fuzzy algorithm for a control system that accepts the structured programming standard. At the output, the program displays numerical values of the process at the current time or optionally graphical representations.

The software implementation of the fuzzy machine can also be based on a parallel algorithm. Parallel computing gives a new dimension to the construction of algorithms and programs. It is emphasized that parallel programming is not a simple extension of serial programming and that not all sequential algorithms can be parallelized.

\section{Design of fuzzy systems with Matlab}

In the Matlab software environment, there are specialized toolboxes for the analysis and design of intelligent control systems, which include the Fuzzy Logic Toolbox (FLT). This toolbox consists of a set of Matlab files (in two subdirectories: FUZZY and FUZZYDEMOS) that allow you to tackle the steps characteristic of synthesizing a fuzzy inference (FIS) based system. The subdirectory FUZZY contains function files divided into the following categories of specific functions and operations:

- Graphical user interface (GUI) functions; functions for editing the fuzzy inference system (FIS), membership functions (FA) and rules used, inference diagrams and associated control 
surfaces; functions for the FIS generation command (by fusing - defining a FA for each variable involved in fuzzy rules, by mitigation based on the estimation of fuzzy inferences, and by transferring parameters between functions and variables, respectively. by generic evaluation of FA and visualization of the control surface); functions for implementation of other routines (FIS of Sugeno type, clusters of C-means type, etc.).

- Operations related to the difference of two FAs with different shapes (sigmoidal, Gaussian, pi, trapezoidal, triangular, Z, S, including their combinations), to concatenate matrices, to discretize the FIS, to evaluate multiple FAs, to edit lines of text, including active auxiliaries.

The FUZZYDEMOS Deputy Director (FUZZY LOGIC TOOLBOX DEMOS) contains several demonstration applications for basic fuzzy functions and operations, as well as complete fuzzy models of intelligent control systems.

Examples of functions and operations:

- Control functions for the graphical user interface with FUZZY for FIS editing

- MFEDIT for editing membership functions

- RULEEDIT for editing SURFVIEW rules viewing control surfaces

- RULEVIEW visualization of rules (RULEVIEW (FIS) and fuzzy inference diagrams for a FIS matrix - RULEVIEW ('FILENAME').

In the construction and simulation of the Fuzzy model for evaluating university teachers, the following steps were:

Step 1: Set up the following diagrams in FIS Editor:

$\checkmark$ block diagram of the fuzzy system for the evaluation of the didactic activity (see Figure 7), which has as variables the following:

- Teaching Method;

- Additional Resources;

- Student Interactions;

- Teaching Skills;

- Implication.

The input variable "Explanations" and its sets are shown in Figure 8.

Figure 7. Printscreen with block diagram of the fuzzy system for the evaluation of the didactic activity.

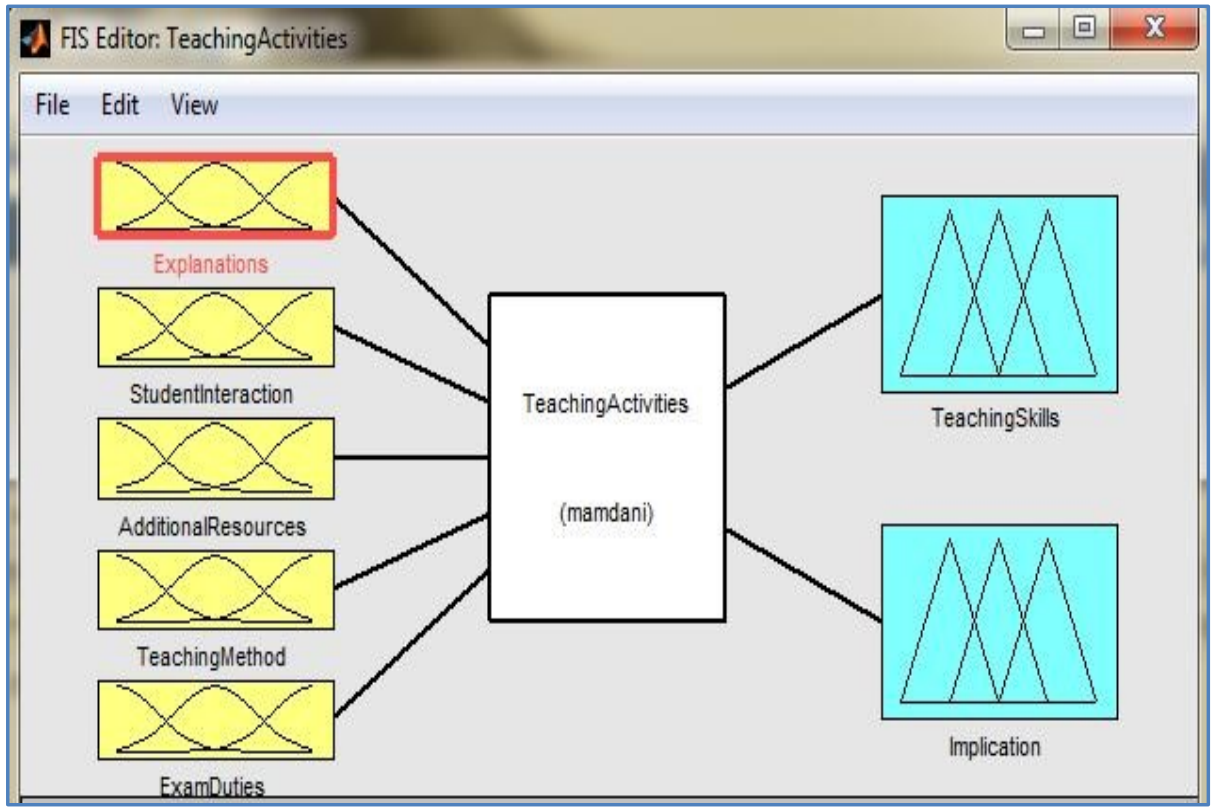


Figure 8. Printscreen with the input variable "Explanations" and its sets.

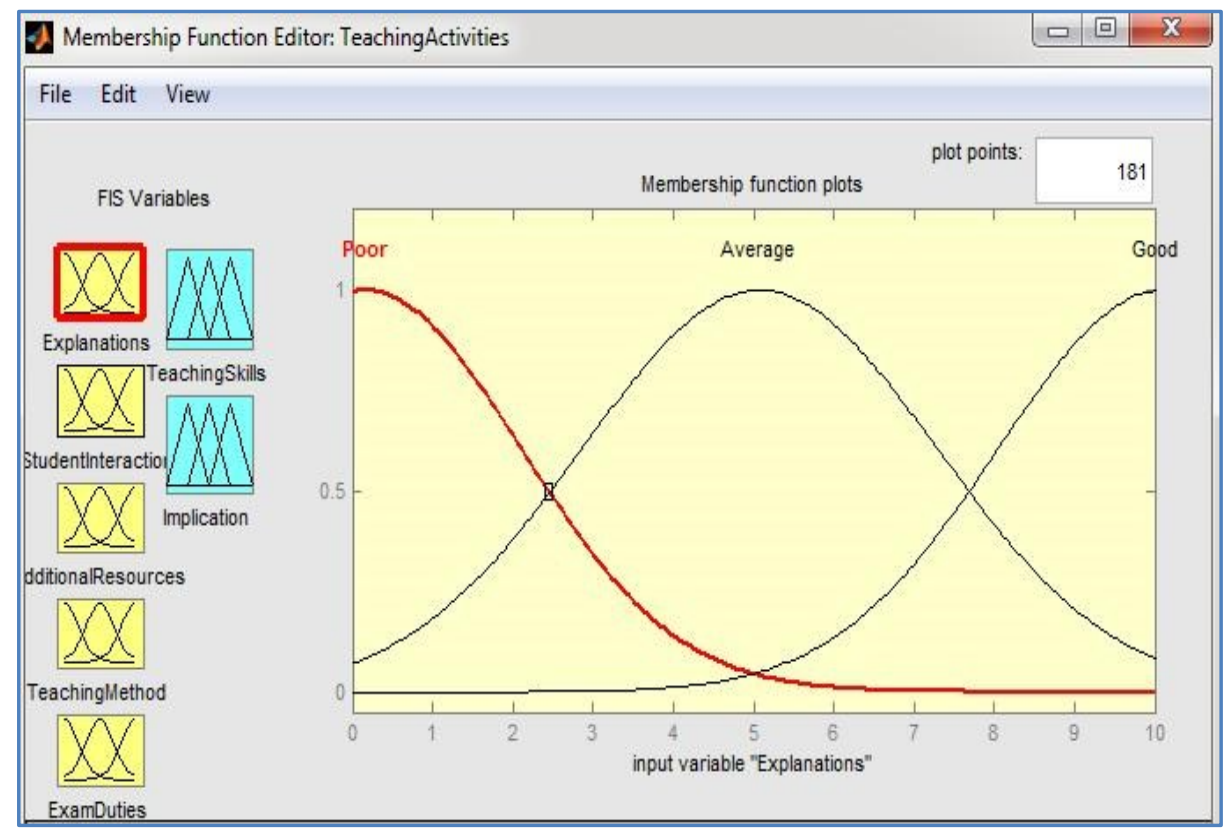

The block diagram of the fuzzy system for the evaluation of the administrative activity (see Figure 9), which has as variables the following:

- Committee Membership;

- Corporate Contribution;

- Co-curricular.

Figure 9. Block diagram of the fuzzy system for evaluating administrative activity.

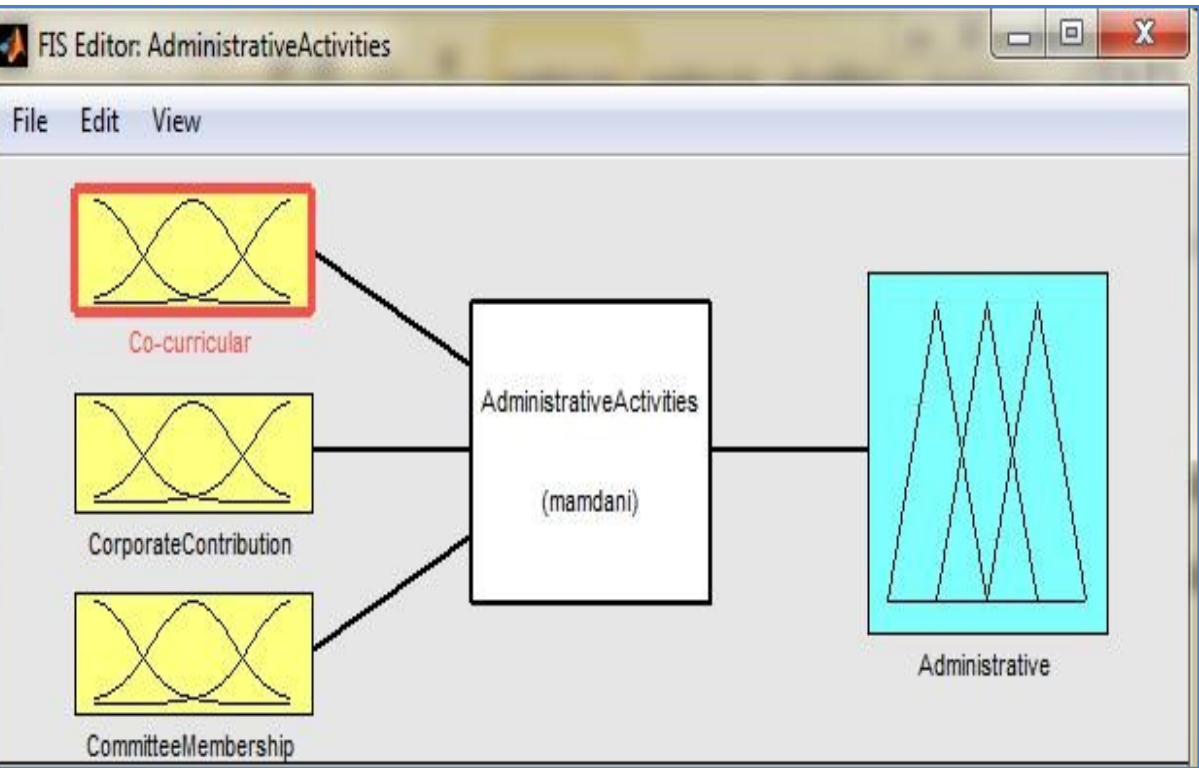

Block diagram of the fuzzy system for evaluating the research activity (Figure 10), which has the following variables:

- Research Projects;

- Research Guidance;

- Book Publications;

- Reserch Paper. 
All these variables underlie the evaluation related to ReasearchSkills and Knowledge Transfer of each university professor (see Figure 10).

Figure 10. Block diagram of the fuzzy system for evaluating the research activity.

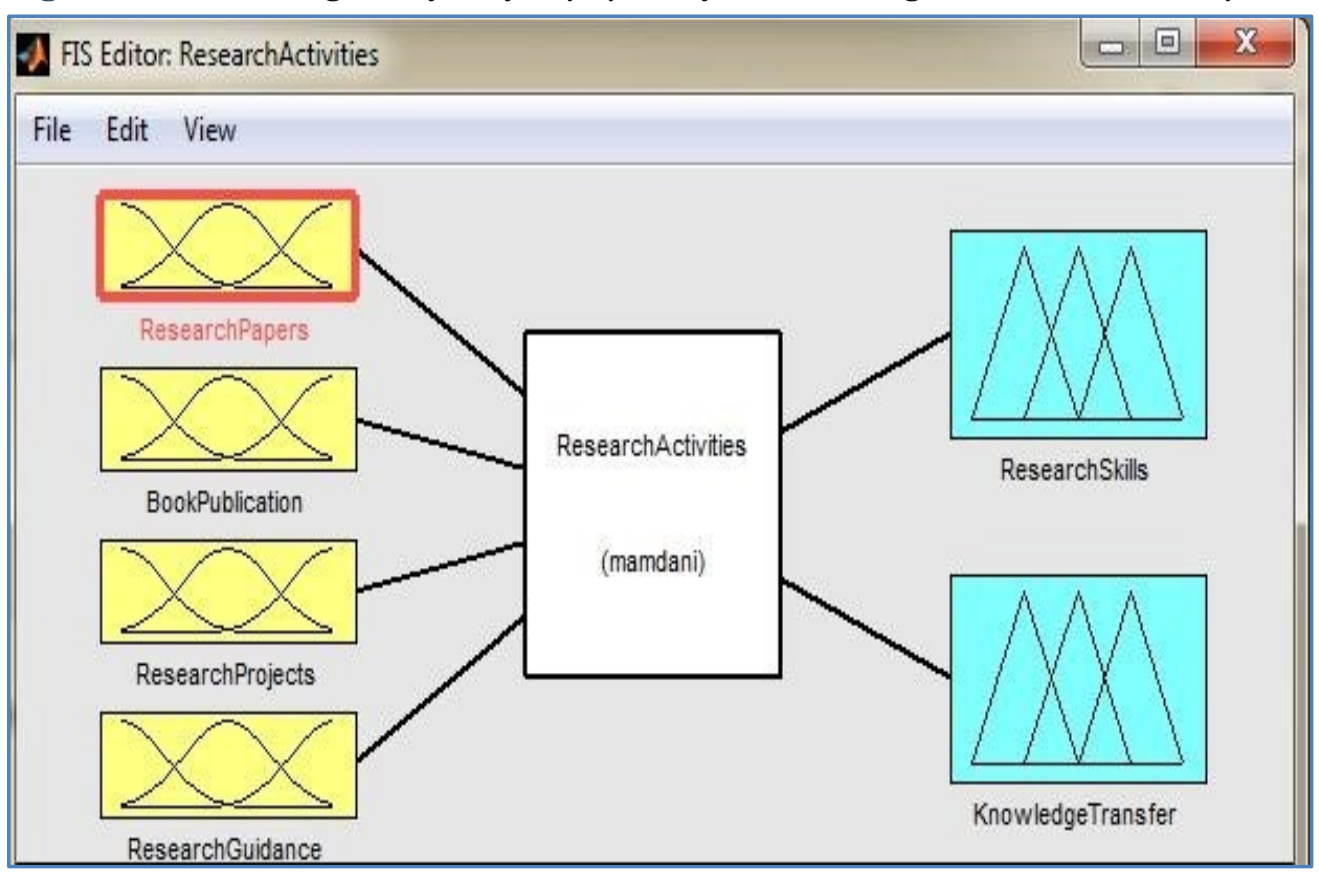

Within the main graphical interface screen (see Figure 11), there is a separation by color code, from top to bottom as follows: blue - data entry, green - results achieved, red - assessment of academics with minimum requirements. The minimum values for each university teaching position used in this simulation are presented within Table 1.

Figure 11. Graphical interface of the evaluation tool.

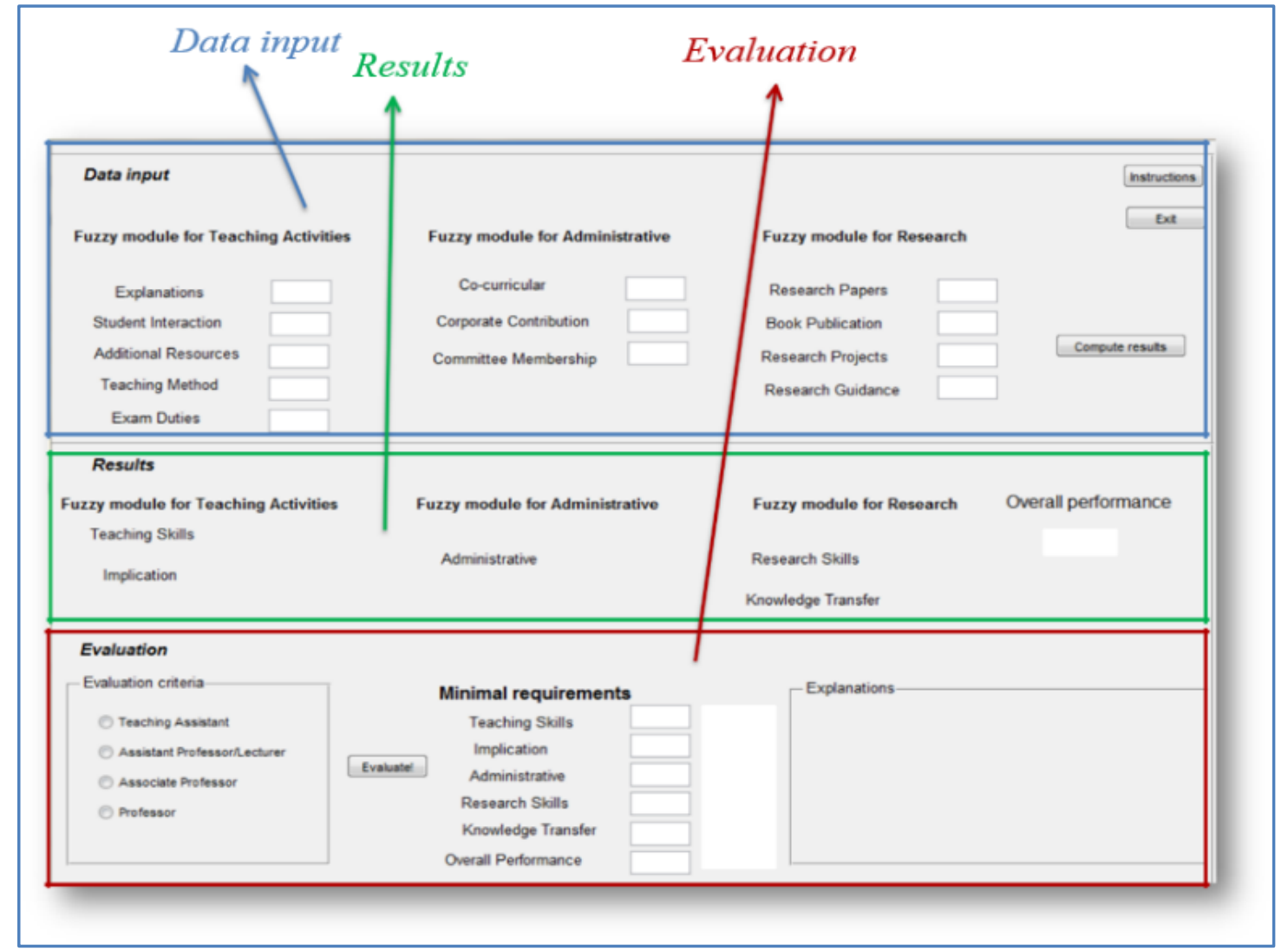


Table 1. Minimum values for each university teaching position.

\begin{tabular}{lcccc}
\hline & Teaching assistant & Assistant professor & Associate professor & Professor \\
\hline Teaching skills & 30 & 40 & 60 & 70 \\
\hline Implication & 30 & 40 & 60 & 60 \\
\hline Administrative & 0 & 25 & 60 & 70 \\
\hline Research skills & 10 & 30 & 60 & 70 \\
\hline Knowledge transfer & 10 & 25 & 60 & 70 \\
\hline Overall performance & 80 & 160 & 300 & 340 \\
\hline
\end{tabular}

Step 2: Simulation of the minimum values for each teaching position university:

In this way, a minimum accepted score was established for each category of university employee: University Professor, Associate University Professor, University Lecturer and University Assistant.

The rule of fuzzy evaluation system for didactic activities (see Figure 12): if (Explanations is average) and (StudentInteraction is good) and (AdditionalResources is high) and (TeachingMethod is average) and (ExamDuties is average) then (TeachingSkills is average) (Implication is good).

Figure 12. The rule of the fuzzy activity evaluation system.

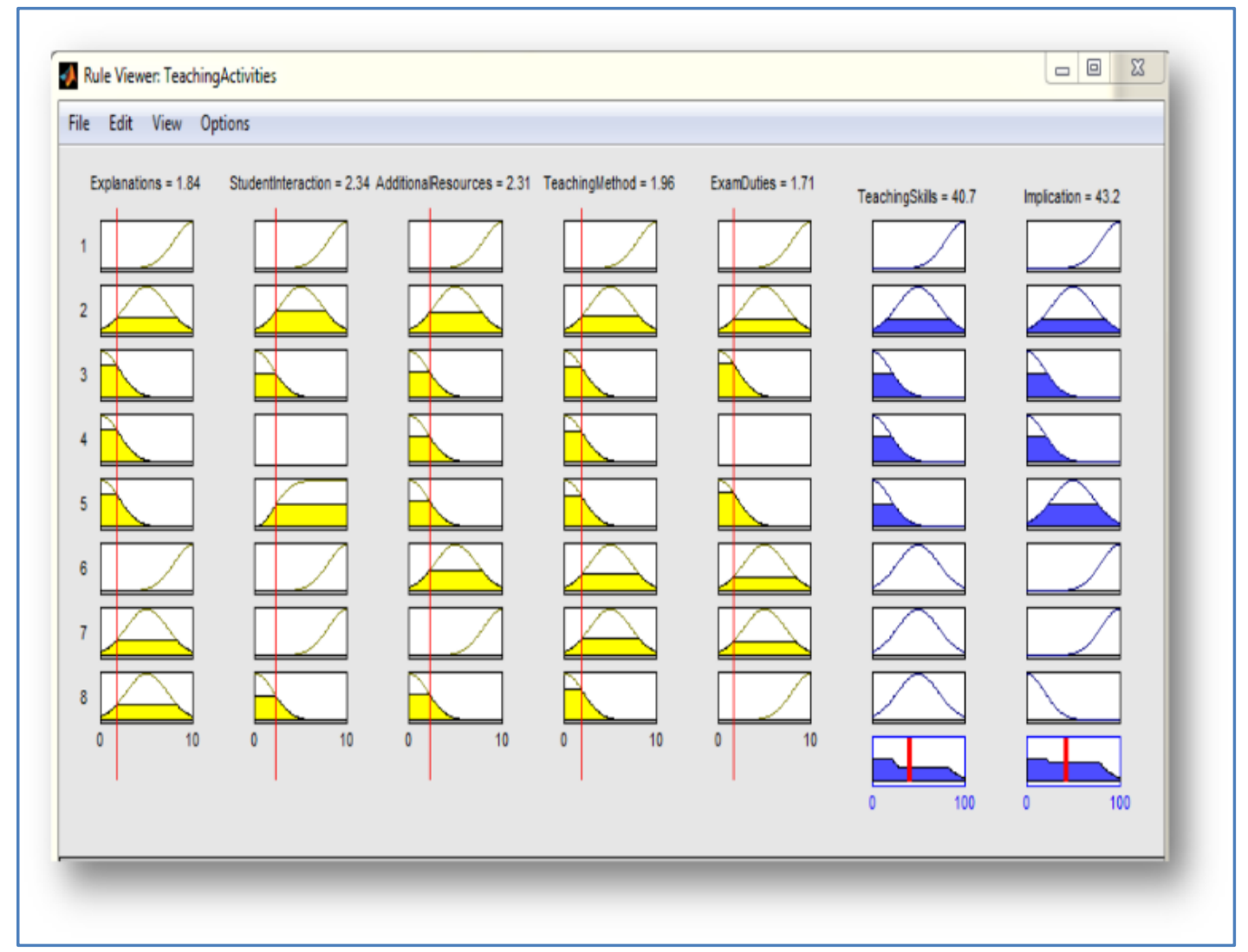

\section{RESULT / FINDINGS}

There are 2 situations with the afferent results of the simulation and validation phase: one in which the minimum conditions are met and the other in which they are not. The print screen in Figure 13 shows us a simulation with the results of the ideal model in which a teaching assistant meets the minimum requirements. Also in this pilot study, a situation was simulated in which a lecturer /assistant Professor does not meet the minimum requirements (see Figure 14). 
Figure 13. Situation when are met the minimum requirements.

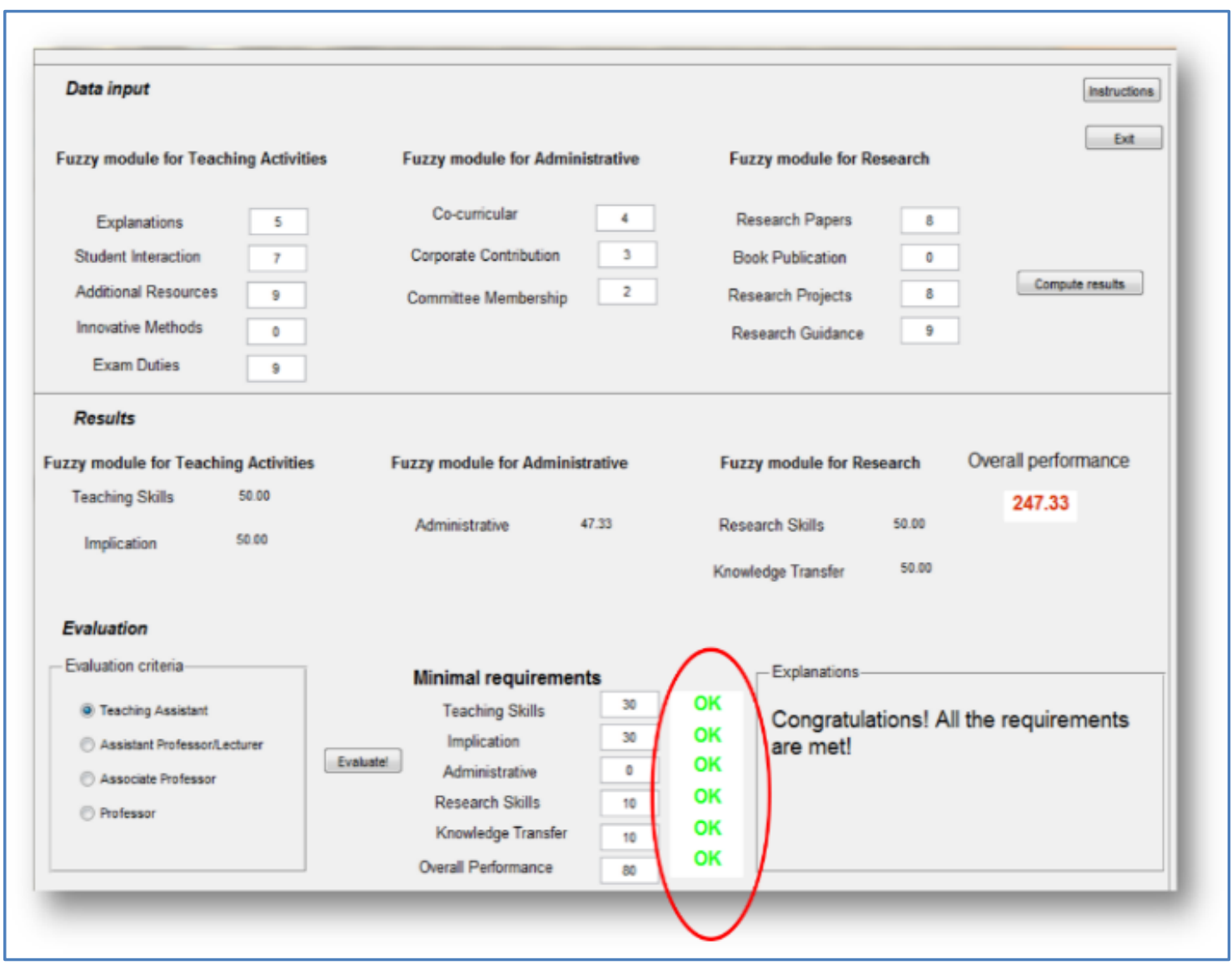

Figure 14. Situation when are NOT met the minimum requirements.

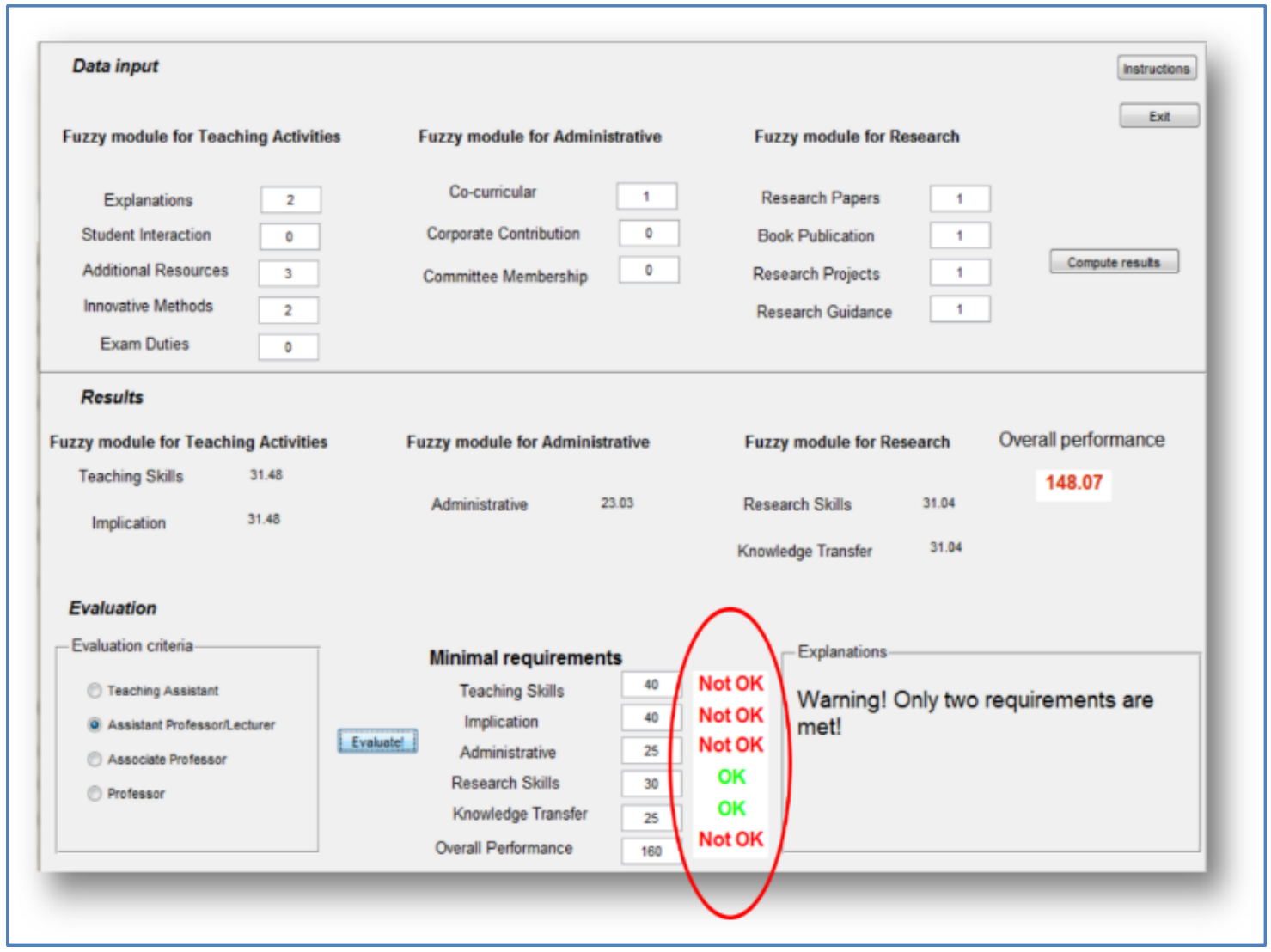




\section{DISCUSSION and CONCLUSION}

Fuzzy set theory is the most general theory of incompleteness yet formulated. Fuzzy logic offers the possibility of reasoning through general knowledge formulated in a general way and has therefore found its application in many fields. Fuzzy concepts and rules can be represented and manipulated by computer, a very valuable feature in the field of knowledge base engineering, where the knowledge of experts is usually formulated in ordinary language.

Applied fuzzy logic is indeed a computational technique that can be used to obtain more meaningful solutions than the classical exact methods when solving specific problems. At the same time, fuzzy systems work very well in the presence of uncertainty, imprecision and "noise". How well such fuzzy systems work is shown by their widespread application in recent years all over the world. There are already a number of well-known applications of fuzzy logic in various fields of science: in automatic control (temperature rules, speed control of the subway, autofocus of video cameras), in shape recognition (fuzzy classification algorithms), in measurements (information processing - sensors), in medicine (control of pacemakers), in economics (fuzzy decision methods).

The model of fuzzy logic applied to the evaluation of university professors is an approximation method that allows to formally model vague "knowledge" stored in a base of rules. The application of fuzzy logic in the evaluation of university personnel at different levels is due to the advantages it offers in the following specific situations:

- Enables modelling of non-linear, complex, or imprecisely known processes for evaluation of university personnel, according to level;

- allows the implementation of the human experience of the evaluators, in this case in the construction of the inference rules, using the linguistic variables explained in the theoretical part..

Fuzzy set theory is used in the evaluation of academics for the following purposes:

- Modeling: in this sense, fuzzy set theory is one of the methods that can be used to model different types of uncertainties related to the teacher's competencies in different circumstances;

- Generalization: classical models and methods are usually based on two-valued logic. This approach often does not adequately represent reality. Fuzzy set theory has been used mainly to relax the classical methods by introducing the gradual character;

- Simplification: fuzzy technology is used to reduce the complexity of data to an acceptable level through linguistic variables or fuzzy analysis

The The present study brought as a novelty the application of fuzzy logic in the field of education, namely in the evaluation of university teachers. The evaluation of university teachers using fuzzy logic is a first stage of quantitative evaluation, which can be the basis for the final evaluation by the heads of departments. Why use fuzzy logic in faculty evaluation? Because it is a helpful complementary tool and has clear advantages such as: it is easy to understand and apply, it is flexible, it is tolerant of imprecise data, it can model complex functions with a high degree of accuracy, it can use expert knowledge, it can be combined with conventional control techniques.

The model of fuzzy logic applied to the evaluation of university professors is an approximation method that can formally model vague "knowledge" stored in a base of rules. The application of fuzzy logic in the evaluation of university personnel at different levels is due to the advantages it offers in the following specific situations:

- Enables modelling of non-linear, complex, or imprecisely known processes for evaluation of university personnel, according to level; 
- allows the implementation of the human experience of the evaluators, in this case in the construction of the inference rules, using the linguistic variables explained in the theoretical part.

The authors consider that the results obtained with the methods proposed in this study can be used in other directions. For example, one of these directions can be the selection of a field of study of universities. In this case, the attitude of students closest to the maximum satisfaction level should be studied.

\section{Declaration of Conflicting Interests and Ethics}

The authors declare no conflict of interest. This research study complies with research publishing ethics. The scientific and legal responsibility for manuscripts published in IJATE belongs to the author(s).

\section{Authorship Contribution Statement}

Authors are expected to present author contributions statement to their manuscript such as; Vasile Florin Popescu: Writing original draft, Methodology, Supervision, and Validation. Marius Sorin Pistol: Resources, Visualization, Software.

\section{ORCID}

Vasile Florin Popescu (DiD http://orcid.org/0000-0002-9972-9904

Marius Sorin Pistol iD https://orcid.org/0000-0003-1172-3637

\section{REFERENCES}

Ahmed, F., \& Toki, M. (2016). A Review on Washing Machine Using Fuzzy Logic Controller. International Journal of Emerging Trends in Engineering, 4(7), 64-67. http://www.wars e.org/IJETER/static/pdf/file/ijeter02472016.pdf

Bellman, R., \& Zadeh, L. (1970). Decision Making in a Fuzzy Environment. Management Sciences, 17(4), 141-164. http://dx.doi.org/10.1287/mnsc.17.4.B141

Chennakesava, R. (2008). Fuzzy logic and neural networks. Basic concepts \& applications, New Age International Publishers, Darya Ganj, New Delhi-110 002, India.

Chuen, L. (1990). Fuzzy Logic in Control Systems: Fuzzy Logic Controller - Part I. IEEE Transactions on Systems, Man, and Cybernetics, 20(2), 404-418. http://ieeexplore.ieee.o $\mathrm{rg} /$ document $/ 52551$

Garrido, L. (2012). A Brief History of Fuzzy Logic. Broad research in artificial intelligence and neuroscience, 3(1), 71-77.

Łukasiewicz, J., \& Tarski, A. (1930). Untersuchungen überden Aussagenkalkül (German). Comptes rendus des séances de la Société des Sciences et des Lettres de Varsovie. CI III, 23, 30-50. English translation: Investigations into the sentential calculus.

Mamdani, E., \& Assilian, S. (1975). An experiment in linguistic synthesis with a fuzzy logic controller. International Journal of Man-Machine Studies, 7(1), 1-13. https://doi.org/10. 1016/S0020-7373(75)80002-2

McCarthy, J. (1959). Programs with Common Sense at the Wayback Machine (archived October 4, 2013). In Proceedings of the Teddington Conference on the Mechanization of Thought Processes, 756-91. Her Majesty's Stationery Office.

Patjoshi R., \& Mohapatra, K. (2010). Experimental Investigation on Microcontroller based Elevator Positioning Control System Using Fuzzy-Logic. International Journal of Advanced Technology and Engineering Exploration, 8(5), 88-94.

Takagi, T., \& Sugeno, M. (1985). Fuzzy Identification of Systems and Its Application to Modeling and Control. IEEE Transactions on Systems, Man, and Cybernetics, SMC-15, 116-132. http://dx.doi.org/10.1109/TSMC.1985.6313399 
Subbulakshmi, L. (2014). Antilock-braking system using fuzzy logic. Middle-East Journal of Scientific Research, 20(10), 1306-1310, 2014, http://dx.doi.org/10.5829/idosi.mejsr.201 $\underline{4.20 .10 .232}$

Vijayana, K., Srivastavaa, P.P., Raghunathb, M.K., \& Saratchandraa, B. (2011) Enhancement of stress tolerance in mulberry. Scientia Horticulturae, 129(4), 511-519. http://dx.doi.or g/10.1016/j.scienta.2011.04.018

Zadeh, L. (1965). Fuzzy sets. Information and Control, 8(3), 338-353. https://doi.org/10.1016 /S0019-9958(65)90241-X

Zadeh, L. (1996). Fuzzy logic=computing with words. IEEE Transactions on Fuzzy Systems, 4(2), 103-111. https://doi.org/10.1109/91.493904

Zadeh, L. (1968). Fuzzy algorithms. Information and Control, 12(2), 94-102. https://doi.org/1 0.1016/S0019-9958(68)90211-8

Zadeh, L. (1971). Quantitative fuzzy semantics. Information Sciences, 3(2), 159-176. https://doi.org/10.1016/S0020-0255(71)80004-X

Zadeh, L. (1973). Outline of a New Approach to the Analysis of Complex Systems and Decision Processes. IEEE Transactions on Systems, Man, and Cybernetics, SMC-3, 28-44. https://doi.org/10.1109/TSMC.1973.5408575 